\title{
Quantum Transport in Networks and Photosynthetic Complexes at the Steady State
}

\author{
Daniel Manzano ${ }^{1,2 *}$
}

1 Instituto Carlos I de Fisica Teorica y Computacional, University of Granada, Granada, Spain, $\mathbf{2}$ Institute for Theoretical Physics, University of Innsbruck, Innsbruck, Austria

\begin{abstract}
Recently, several works have analysed the efficiency of photosynthetic complexes in a transient scenario and how that efficiency is affected by environmental noise. Here, following a quantum master equation approach, we study the energy and excitation transport in fully connected networks both in general and in the particular case of the Fenna-MatthewOlson complex. The analysis is carried out for the steady state of the system where the excitation energy is constantly "flowing" through the system. Steady state transport scenarios are particularly relevant if the evolution of the quantum system is not conditioned on the arrival of individual excitations. By adding dephasing to the system, we analyse the possibility of noise-enhancement of the quantum transport.
\end{abstract}

Citation: Manzano D (2013) Quantum Transport in Networks and Photosynthetic Complexes at the Steady State. PLoS ONE 8(2): e57041. doi:10.1371/ journal.pone.0057041

Editor: Gerardo Adesso, University of Nottingham, United Kingdom

Received June 22, 2012; Accepted January 17, 2013; Published February 26, 2013

Copyright: (c) 2013 Daniel Manzano. This is an open-access article distributed under the terms of the Creative Commons Attribution License, which permits unrestricted use, distribution, and reproduction in any medium, provided the original author and source are credited.

Funding: The research was funded by the Austrian Science Fund (FWF): F04011 and F04012 and by Spanish MEC-FEDER, project FIS2009-08451, together with the Campus de Excelencia Internacional and the Junta de Andalucia, project FQM-165 (Spain). The funders had no role in study design, data collection and analysis, decision to publish, or preparation of the manuscript.

Competing Interests: The author has declared that no competing interests exist.

* E-mail: daniel.manzano@uibk.ac.at

\section{Introduction}

In the last years, quantum transport in photosynthetic complexes has become an interesting field of study and debate. An important part of this research focusses on the excitation transfer from the antennae that harvest the sunlight to the reaction centre $(\mathrm{RC})$ where the photosynthetic process takes place. More concretely, for the Fenna-Matthew-Olson (FMO) complex of green sulfur bacteria, empirical evidence suggests that such transport is coherent even at room temperature [1-3]. These experiments show that the transient behaviour takes place on time scales much shorter than the decoherence time due to the environment. Thus, most of the recent analysis has focussed on the single-excitation scenario in the transient regime obtained after pulsed photoexcitation [4-12].

Actually, there is a vivid debate about the validity of the singleexcitation picture for modelling the photosynthetic process in vivo. Photosynthesis in nature is a continuous process of absorption of energy from a radiation field. As there is no specific measurement mechanism that determines when the quanta of energy are effectively absorbed, some authors have argued that the photosynthetic complex and the radiation field should evolve to a steady state where the energy is constantly flowing through the system $[13,14]$. Some of the conclusions of [14], regarding the importance of a steady state picture, are summarized in the following paragraph: 'The classical picture of the photon as a particle incident on the molecule, repeatedly initiating dynamics, also assumes a known photon arrival time. This too is incorrect and inconsistent with the quantum analysis insofar as no specific arrival time can be presumed unless the experiment itself is designed to measure such times'. Also, it has been shown that some conclusions regarding the presence of entanglement in this kind of system rely on the assumption that the system is excited by a single excitation Fock state. This state cannot be obtained just by weak illumination, and changing this assumption for a more realistic one changes dramatically the conclusions [15]. These arguments makes it reasonable to analyse the natural photosynthetic processes also in other regimes, such as a steady state scenario.

Moreover, quantum transport in a non-equilibrium steady state is an active field in condensed matter physics. For ordered systems composed of qubits or harmonic oscillators, it has been shown that it is possible to violate Fourier's law and thus achieve an infinite thermal conductivity in the absence of noise $[16,17]$. This ballistic transport turns into a diffusive one, with finite conductivity, if noise is added to the system as a dephasing channel, reducing therefore the energy transfer. That fact highlights the importance of the interaction with a dephasing environment for the energy transfer. The analysis of quantum transport can contribute to the design of artificial light-harvesting systems that are more efficient and robust [18].

Recently, quantum transport in photosynthetic complexes has been analysed through different models with different measures of the efficiency, principally in the single-excitation regime. In [10], the dynamics of the FMO complex was analysed by the use of a Markovian Redfield equation and by a generalized BlochRedfield equation [19]. The measure of efficiency that they use is the average time that a single excitation spends in the network before being absorbed by the sink. The results show that the Redfield approach correctly describes the dynamics of the system, but also that it fails to determine the optimal dephasing ratio that minimizes the trapping time. Moreover, this approach gives the unphysical results of a zero trapping time in the limit of strong dephasing $\gamma \rightarrow \infty$. An analogous model was considered in [9], with the difference that the efficiency was quantified by the population 
of the sink in the long time limit. Finally, Scholak et al. [11,12] have studied this problem in the absence of a sink, in such a way that the only incoherent dynamics was due to the presence of a dephasing environment. Here, the index for the quantification of the efficiency was the highest probability of finding the excitation in the outgoing qubit in a time interval $[0, \tau]$, with $\tau$ being related to the estimate of the duration of the excitation transfer in real systems. The authors conclude that the addition of noise can increase the efficiency, but mainly in configurations that initially performed poorly. Despite their differences, all these papers coincide in analysing only the transient behaviour, and not the steady state, and they use very different indexes for quantifying the efficiency of the system.

In this paper, we analyse the energy transfer in quantum networks and, specifically, in the FMO complex in a steady state. We show that the excitations move coherently through the system also in this regime. The addition of a dephasing environment reduces, but does not destroy, the coherent transport. We also analyse the change in efficiency due to such an environment. The model we consider here is based on a quantum network connected to a thermal bath, to model the absorption of energy from the radiation field, and to a sink, that delivers the energy quanta to the reaction centre. As a particular case, we analyse the FMO complex and similar fully connected networks. In this scenario, the system evolves to a non-equilibrium steady state, where all the observables remain constant. A similar framework has already been used to analyse entanglement in light-harvesting complexes in the transient regime [20].

As has been discussed before, several indexes of the efficiency are usually applied in order to calculate the efficiency of these kinds of system. Also, for the complete photosynthetic procces itself, there is an important difference between analysing it by the use of quantum efficiency, that is, the average number of absorbed photons that finally give rise to photosynthetic products, and the energy efficiency. The second one is considered a more appropriate measure for comparing the efficiency of photosynthetic complexes with artificial light harvesting systems and for analysing the global procedure [18]. Because of that, we will use the energy transfer per unit of time, that is, the power, as our principal index of the efficiency of the systems. This measure will be compared with the excitation transfer, which corresponds to the quantum efficiency. We will show that, in general, they behave in a very different way, especially under the effect of noise.

The present paper is organized as follows. In the next section, we introduce the details of the model and of the master equation which describes its dynamics. In Section III, we introduce two indexes for evaluating the efficiency, and we perform an analytical comparison between the two of them. Uniform and general networks are analysed in Section IV, while in Section V we focus our attention on the FMO complex and related Hamiltonians. Finally, in Section VI some conclusions are drawn.

\section{Methods}

\section{Description of the Model and the Master Equation}

The energy transfer in photosynthetic complexes, such as the FMO complex, can be described by exciton dynamics. Such systems can be modelled as fully connected networks of two-level systems (qubits), and several recent works have analysed photosynthetic processes by the use of this framework. Most of these works have used a single excitation framework, different from the one used here. Since the FMO complex is composed of seven chromophores, it should be modelled by a network of seven qubits. To describe the absorption of energy from the antennae and the decay to the reaction centre $(\mathrm{RC})$, we use a Markovian quantum master equation in Lindblad form [21]. The validity of this master equation has been numerically verified in systems composed of harmonic oscillators [22], showing that it is accurate for small coupling even in the low and high temperature regimes. In the transient regime, similar master equations have been previously used to describe the dynamics of the FMO complex and to analyse the effects of noise on the quantum dynamics $[9,10]$.

The quantum evolution of the network is determined by a Hamiltonian of the form

$$
H=\sum_{i=1}^{7} \hbar \omega_{i} \sigma_{i}^{+} \sigma_{i}^{-}+\sum_{\substack{i, j=1 \\ j \neq i}}^{7} \hbar g_{i j}\left(\sigma_{i}^{+} \sigma_{j}^{-}+\sigma_{i}^{-} \sigma_{j}^{+}\right),
$$

where $\sigma_{i}^{ \pm}$are the raising and lowering operators that act on qubit $i, \hbar \omega_{i}$ are the one-site energies, and $g_{i j}$ represents the coupling between qubits $i$ and $j$.

The interaction of the photosynthetic complex with the environment can be divided into four different processes, and each of them is modelled by a different Lindblad superoperator. According to the empirical results from [23], the absorption of energy from the antennae populates principally site 1 , with a nonnegligible population of site 6. For simplicity, this process is modelled in our paper by a thermal bath connected to site 1 . The delivery of the excitation energy from the complex to the $\mathrm{RC}$ is mediated by site 3 . As this is a irreversible process, we model it by a sink, a zero temperature thermal bath, which removes the excitations from this site in an incoherent way. Also, photosynthetic complexes are not isolated from the surrounding environment. They interact with other biological components, an interaction that in vivo happens at room temperature. That leads to two different effects on the system. First, the loss of coherence in the transport due to the dephasing induced in the system, and second, the absorption of excitations by the environment.

The injection of excitations by the thermal bath acting on the first qubit is modelled by the Linblad superoperator

$$
\begin{aligned}
& \mathcal{L}_{\text {th }} \rho=\Gamma_{\text {th }}(n+1)\left(\sigma_{1}^{-} \rho \sigma_{1}^{+}-\frac{1}{2}\left\{\sigma_{1}^{+} \sigma_{1}^{-}, \rho\right\}\right) \\
& +\Gamma_{\text {th }} n\left(\sigma_{1}^{+} \rho \sigma_{1}^{-}-\frac{1}{2}\left\{\sigma_{1}^{-} \sigma_{1}^{+}, \rho\right\}\right),
\end{aligned}
$$

where the parameter $\Gamma_{\text {th }}$ represents the strength of the coupling between the quantum system and the environment. As there are no empirical estimates of this parameter, we take $\Gamma_{\mathrm{th}}=1 \mathrm{~cm}^{-1}$ through the paper. The parameter $n$ is the mean number of excitations with frequency $\omega$ in the bath.

The delivery of energy from the system to the RC is modelled by a second Lindblad superoperator, which models a zero temperature thermal bath. This bath is usually referred to as a sink.

$$
\mathcal{L}_{\text {sink }} \rho=\Gamma_{\text {sink }}\left(\sigma_{3}^{-} \rho \sigma_{3}^{+}-\frac{1}{2}\left\{\sigma_{3}^{+} \sigma_{3}^{-}, \rho\right\}\right)
$$

This term describes the irreversible decay of the excitations to the reaction centre. When the excitation is absorbed by the sink, it triggers a charge separation event and can not go back to the complex. We assume that this process is faster than the system dynamics and, because of that, the sink does not saturate. So, it 
can be described by a Markovian approach. Again, the coupling strength $\Gamma_{\text {sink }}$ has not been estimated from experimental data, and we choose $\Gamma_{\text {sink }}=1 \mathrm{~cm}^{-1}$.

The interaction between the complex and its surrounding environment has two different effects. First, it reduces the quantum coherence of the system. This is modelled in our master equation by the term

$$
\mathcal{L}_{\mathrm{deph}} \rho=\gamma \sum_{i=1}^{7}\left(\sigma_{i}^{+} \sigma_{i}^{-} \rho \sigma_{i}^{+} \sigma_{i}^{-}-\frac{1}{2}\left\{\sigma_{i}^{+} \sigma_{i}^{-}, \rho\right\}\right) .
$$

This interaction does not change the mean number of excitations in the system but, as we will see in next section, it can affect the its mean energy, and so the energy flux. Also, this is the interaction that can improve the efficiency of the system by removing the destructive coherences that delay the transmission of the excitation to the third site. The parameter $\gamma$ represents the strength of the interaction between the complex and the dephasing environment. This will be the free parameter we use in this paper in order to optimize the efficiency of the system.

Finally, the system is also susceptible to a radiative decay process that transfers the excitations from the complex to the environment. This process effectively reduces the mean number of excitations in the system together with the mean energy.

$$
\mathcal{L}_{\mathrm{diss}} \rho=\Gamma_{\mathrm{diss}} \sum_{i=1}^{7}\left(\sigma_{i}^{-} \rho \sigma_{i}^{+}-\frac{1}{2}\left\{\sigma_{i}^{+} \sigma_{i}^{-}, \rho\right\}\right) .
$$

Again, the coupling parameter $\Gamma_{\text {diss }}$ has not been inferred from experimental data, so we will check different values of it in order to analyse the noise-enhancement under different kinds of dissipative environments.

The complete time evolution of the density matrix of the system is described by the master equation

$$
\dot{\rho}=-\frac{i}{\hbar}[H, \rho]+\mathcal{L}_{\text {th }} \rho+\mathcal{L}_{\text {sink }} \rho+\mathcal{L}_{\text {deph }} \rho+\mathcal{L}_{\text {diss }} \rho
$$

The steady state occurs in the long time limit, and satisfies the condition $\dot{\rho}=0$, meaning that the density matrix is stationary.

Our analysis has been performed in two different regimes, depending on the rate of excitation injection. First, we study the low-temperature case, by choosing $n=1$. This choice corresponds to a slow injection of excitations into the system, as should be the case for the FMO complex under weak illumination. In this case, the injection of energy is so slow that the probability of finding more than one excitation at the same time in the network is almost negligible (but not excluded). Second, we simulate a high temperature environment, $n=100$, where there is a higher probability of finding more than one excitation inside the system at the same time. As we will see in the following sections, these different situations lead to different results.

\section{Energy and Excitation Fluxes}

In order to evaluate the efficiency of these systems, we consider two different indexes. First, we observe that the net energy transfer across the system is quantified by the time derivative of the expectation value of the Hamiltonian,

$$
\dot{E}=\frac{d}{d t}\langle H\rangle=\operatorname{Tr}(H \dot{\rho})
$$

By using the master equation (6) and the fact that, in the steady state, the mean energy of the system is conserved, we obtain an expression for the energy exchanged through the different environmental channels.

$$
\begin{aligned}
& 0=\operatorname{Tr}\left(H \mathcal{L}_{\text {th }} \rho\right)+\operatorname{Tr}\left(H \mathcal{L}_{\text {sink }} \rho\right)+\operatorname{Tr}\left(H \mathcal{L}_{\text {deph }} \rho\right)+\operatorname{Tr}\left(H \mathcal{L}_{\text {diss }} \rho\right) \\
& =: J_{\text {th }}+J_{\text {sink }}+J_{\text {deph }}+J_{\text {diss }}
\end{aligned}
$$

where $J_{\text {th }}$ represents the energy flux from the thermal environment to the system, $J_{\text {sink }}$ from the system to the sink, $J_{\text {deph }}$ between the system and the dephasing environment, and $J_{\text {diss }}$ is the energy loss due to the decay of the excitations.

As our main interest is to quantify the energy that flows from the system to the sink, we use $J \equiv J_{\text {sink }}$ as our first index to measure the efficiency. This expression has been applied in previous papers in order to analyse the efficiency of quantum refrigerators [25] and to study Fourier's law in quantum systems [16,17].

The second measure of the efficiency that we use is the excitation flux, defined as the number of excitations incoherently absorbed by the sink per unit time. For a time interval $[0, \tau]$, it is given by

$$
p_{\text {sink }}=\Gamma_{\text {sink }} \int_{0}^{\tau} P d t
$$

where $P \equiv\left\langle\sigma_{3}^{+} \sigma_{3}^{-}\right\rangle$is the population of the third site, obviously time-independent in the steady state. This fact allows us to consider the population of the third site as a measure of the speed of the excitation transfer to the RG. These two measures, the energy transfer and the population of the third site, are not equivalent since they measure slightly different quantities. Indeed, the results about the efficiency of the systems are different depending on the measure that is used.

In order to relate these two quantities, it is is useful to decompose the Hamiltonian:

$$
H=H_{3}+\sum_{\substack{j=1 \\ j \neq 3}}^{7} H_{3 j}+\tilde{H}
$$

where $H_{3}=\hbar \omega_{3} \sigma_{3}^{+} \sigma_{3}^{-}, \quad H_{3 j}=\hbar g_{3 j}\left(\sigma_{3}^{+} \sigma_{j}^{-}+\sigma_{3}^{-} \sigma_{j}^{+}\right)$, and $\tilde{H}$ represents the part of the Hamiltonian that does not involve qubit three. Hence, $J$ can be expressed by

$$
J=\operatorname{Tr}\left(H_{3} \mathcal{L}_{\text {sink }}\right)+\sum_{\substack{j=1 \\ j \neq 3}}^{7} \operatorname{Tr}\left(H_{3 j} \mathcal{L}_{\text {sink }}\right)+\operatorname{Tr}\left(\tilde{H} \mathcal{L}_{\text {sink }}\right),
$$

and the last term is null due to the fact that $\operatorname{Tr}\left(\mathcal{L}_{\text {sink }}\right)=0$. A straightforward evaluation of this expression allows of expressing the energy transfer to the sink as a function of the population of the third site and the coherences between this qubit and all the others. 
$J=-\Gamma_{\text {sink }} \hbar\left(-\omega_{3} P-\sum_{\substack{i=1 \\ i \neq 3}}^{7} \frac{g_{i 3}}{2}\left(\left\langle\sigma_{3}^{+} \sigma_{i}^{-}\right\rangle+\left\langle\sigma_{i}^{-} \sigma_{3}^{+}\right\rangle\right)\right)$.

As $\left\langle\sigma_{3}^{+} \sigma_{i}^{-}\right\rangle=\left\langle\sigma_{3}^{-} \sigma_{i}^{+}\right\rangle^{*}$, the heat transfer will depend only on the real part of the next-neighbours coherences. It has been proved that in a linear chain, with equal one-site energies and couplings, these coherences are purely imaginary and the heat flux depends only on the population [16]. In the general case, these coherences will be nonzero and they can contribute in a positive or negative way to the energy flux. It is clear from Eq. (12) that there is a strong connection between the energy and the population fluxes. It is also clear that these measures are related but not equivalent.

In a similar way, the expression of the energy flux due to the dephasing environment can be calculated

$$
J_{\mathrm{deph}}=\sum_{\substack{i, j=1 \\ j>i}}^{7}-\frac{\gamma \hbar g_{i j}}{2}\left(\left\langle\sigma_{i}^{-} \sigma_{j}^{+}\right\rangle+\left\langle\sigma_{i}^{+} \sigma_{j}^{-}\right\rangle\right)
$$

Again, in the concrete case of a uniform chain, these nextneighbours coherences are purely imaginary and because of that this term vanishes. In a general fully connected network, these elements are in general complex and there is an interchange of energy between the network and the environment due to the dephasing channel. That effect happens because the environment projects the system onto a basis that is not composed of the Hamiltonian's eigenvectors. That means a reduction of the elements that are not eigenvectors of a single site basis. As the eigenvalues of the Hamiltonian are usually composed of these nonlocal terms, this interaction can effectively reduce or increase the energy inside the system. Recently, the energy cost of quantum projective measurements has been analysed and related to the work value of the acquired information [24]. Measurements can change the energy of the network and, in the steady state, that will lead to an energy flux. In a similar way, the presence of dissipation in the system reduces both the excitation and energy fluxes.

\section{Results and Discussion}

\section{General Networks}

First, we analysed the case of a general fully connected network where the excitations are injected by a thermal reservoir and delivered to a sink. For that, we have calculated the energy end excitation rates as functions of the dephasing ratio for a fully connected homogeneous network in both the low and high temperature regime. Both the one-site energies and the couplings between the qubits are equal and they have the value $\hbar \omega_{i}=\hbar g_{i j}=1 \mathrm{~cm}^{-1} \forall i, j$. The couplings with the thermal environment and the sink are $\Gamma_{\mathrm{th}}=\Gamma_{\text {sink }}=1 \mathrm{~cm}^{-1}$. The analysis is made for different values of the dissipation rate $\Gamma_{\text {diss }}$, in order to analyse the effects of a dissipative environment on the transfer.

The results for a low temperature thermal bath $(n=1)$, and several dissipation rates $\left(\Gamma_{\text {diss }}=0,0.2,0.5,1 \mathrm{~cm}^{-1}\right)$, are shown in Fig. 1. The two different measures of efficiency, the energy and excitation transfer, exhibit very different behaviours when the dephasing parameter increases. The excitation transfer increases for small values of the dephasing ratio and decreases for higher ones. It is clear that the excitation transfer at the steady state can be improved by the addition of external noise to the system. This effect is due to the reduction of destructive interferences that inhibit the transport of the excitation to the qubit coupled to the sink, as is explained in [9]. Similar results have been obtained in the transient regime [9]. On the other hand, the energy transfer is always reduced if a dephasing environment acts on the system. That means that, even where the noise can enhance the number of particles arriving at the sink per unit time, it can at the same time reduce the energy transferred to it. This difference is due to the reduction of the coherences in Eq. (12). For high values of the dephasing rate $\gamma$, the transport is reduced, due to a quantum Zeno effect, that avoids the coherent transport. The optimal dephasing ratio that maximizes the excitation transfer is $\gamma_{\text {uniform }}^{*}=1.25 \pm 0.1 \mathrm{~cm}^{-1}$. For the energy transfer, the optimal rate is to have no dephasing at all.

For a high temperature bath $(n=100)$, we have similar results, as is displayed in Fig. 2. For this case, both fluxes are higher than in the low temperature regime, as is to be expected, but the effect of dissipation is different. If the system is under the effect of a highly dissipative environment, as $\Gamma_{\text {diss }}=1 \mathrm{~cm}^{-1}$, the efficiency of the excitation flux can not be improved by the addition of dephasing. The optimal dephasing ratio is independent of the temperature of the bath for no dissipation, and in the low temperature regime it is independent of the dissipation. That indicates that it could be a general property of the Hamiltonian. For a highly dissipative bath, the noise-enhancement of the excitation transfer progressively disappears and, because of that, there is no optimal dephasing rate.

For analysing a more general case, we have also generated 7000 random Hamiltonians, by Monte Carlo simulation, where the onesite energies and the couplings between the qubits are randomly selected from a uniform distribution, with $\hbar \omega_{i} \in[0,10]$ and $\hbar g_{i j} \in[-10,10] \mathrm{cm}^{-1}$. Again, the heat and the excitation transfer exhibit very different behaviours. In Fig. 3, the heat and population fluxes are plotted for a small dephasing ratio, $\gamma=1$, as a function of the fluxes for the same systems without dephasing, in the low temperature regime. For the heat transfer, the addition of noise to the system can either increase or decrease the efficiency of the system. This improvement is smaller when the system is highly efficient. That shows that the most efficient configurations are also the most robust against the addition of noise, and they are very difficult to improve. The excitation transfer to the sink is improved for all the analysed Hamiltonians, again this improvement is less when the efficiency of the system is greater. These results are compatible with the conclusions of [11], where it is shown that in the single excitation picture, systems with low efficiency are more suitable for improvement by a dephasing channel than are the highly efficient ones.

The results in the case of a high energy thermal bath are very similar, as is displayed in Fig. 4. There, the enhancement is smaller than for $n=1$ and the population transfer can also be reduced, but only to a small degree. That implies that the energy transfer is more stable under the effects of noise for systems with high number of excitations than for ones in which this number is smaller. Again, the most efficient configurations are more robust against the effect of noise.

Both in the low and high temperature regimes, the effects of dissipation in these simulations are similar. The presence of dissipation reduces both the fluxes and the improvement possible with a dephasing noise. The results are very similar to the ones displayed in Figs. 3 and 4 and are not shown for simplicity.

The differences between the energy and heat transfer come from the fact that the energy transfer depends both on the population of the outgoing site and the coherences between it and the others qubits. Even if the dephasing channel increases the population of 

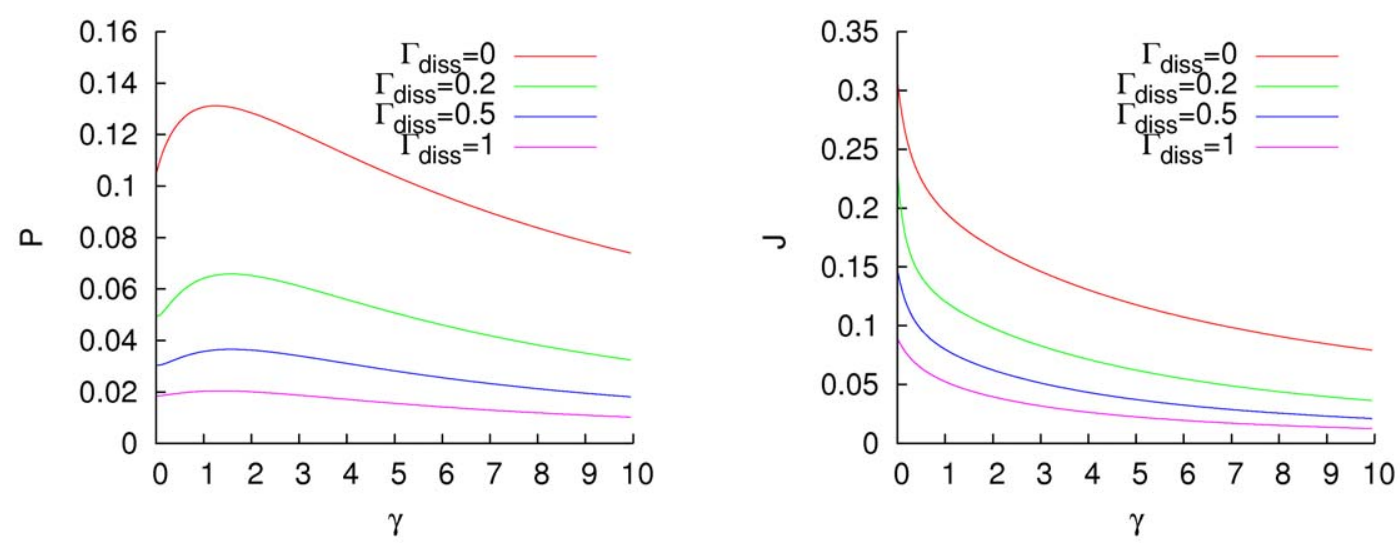

Figure 1. Excitation (left) and energy (right) fluxes for a homogeneous fully connected network, with $\hbar \omega_{i}=\hbar g_{i j}=1 \mathrm{~cm}^{-1} \forall i, j$, as functions of the dephasing ratio $\gamma$, for a low-temperature thermal bath, $n=1$, and different dissipation rates $\Gamma_{\text {diss }}$ Units of $\mathrm{cm}^{-1}$. doi:10.1371/journal.pone.0057041.g001

this qubit, increasing consequently the transfer of excitations to the sink, it also reduces the amount of coherence between qubits. These two effects compete to determine whether the energy transfer will be improved or depressed. The relation between the one-site energies and the couplings play an essential and non-trivial role in this effect.

\section{Photosynthetic Complexes}

To analyse light-harvesting biological systems, we study the FMO protein complex in green sulfur bacteria. This complex is assumed to have seven chromophores and, because of that, it can be modelled as a network of seven sites. We use the experimental Hamiltonian given in [23], tables $2 \mathrm{MEAD}$ and 4 (trimer). $\mathrm{In}^{-1}$, this Hamiltonian reads:

$$
\begin{aligned}
& H_{\mathrm{FMO}}= \\
& \left(\begin{array}{ccccccc}
12445 & -104.0 & 5.1 & -4.3 & 4.7 & -15.1 & -7.8 \\
-104.0 & 12450 & 32.6 & 7.1 & 5.4 & 8.3 & 0.8 \\
5.1 & 32.6 & 12230 & -46.8 & 1.0 & -8.1 & 5.1 \\
-4.3 & 7.1 & -46.8 & 12355 & -70.7 & -14.7 & -61.5 \\
4.7 & 5.4 & 1.0 & -70.7 & 12680 & 89.7 & -2.5 \\
-15.1 & 8.3 & -8.1 & -14.7 & 89.7 & 12560 & 32.7 \\
-7.8 & 0.8 & 5.1 & -61.5 & -2.5 & 32.7 & 12510
\end{array}\right)
\end{aligned}
$$

As for this Hamiltonian the one-site energies are two orders of magnitude higher than the couplings, we can expect that they should play a more relevant role for the energy flux. In this concrete case, the energy and excitation transfer are very similar, in contrast to the general networks analysed before. As there are no empirical measurements of the coupling between the complex and the antennae or the RC, we choose $\Gamma_{\text {th }}=\Gamma_{\text {sink }}=1 \mathrm{~cm}^{-1}$.

In Fig. 5 the energy and excitation fluxes are plotted as a function of the dephasing ratio $\gamma$, for $n=1$, and $\Gamma_{\text {diss }}=0,0.2,0.5,1 \mathrm{~cm}^{-1}$. The addition of noise improves both the excitation and the energy fluxes in this system and both measures of efficiency have practically the same behaviour. Similar results arise for $n=100$, and are omitted. The optimal value of the dephasing ratio is equal for both the less and the highly excited scenarios, and for the energy and excitation transfers, with an optimal value $\gamma_{\mathrm{FMO}}^{*}=60 \pm 1 \mathrm{~cm}^{-1}$. It is also independent of the dissipation acting on the system. This result is of the same order, but quantitatively different, as the one obtained in [10] by using the mean trapping time as a measure of the efficiency and a global Redfield equation to describe the dynamics of the network. This optimal ratio is higher than in the case of the homogeneous network analysed before, due to the different order of magnitude of the ratio of the energies and the couplings. Again, the presence of dissipation in the system reduces the fluxes but it does not affect the qualitative behaviour. The similarity between both measures of
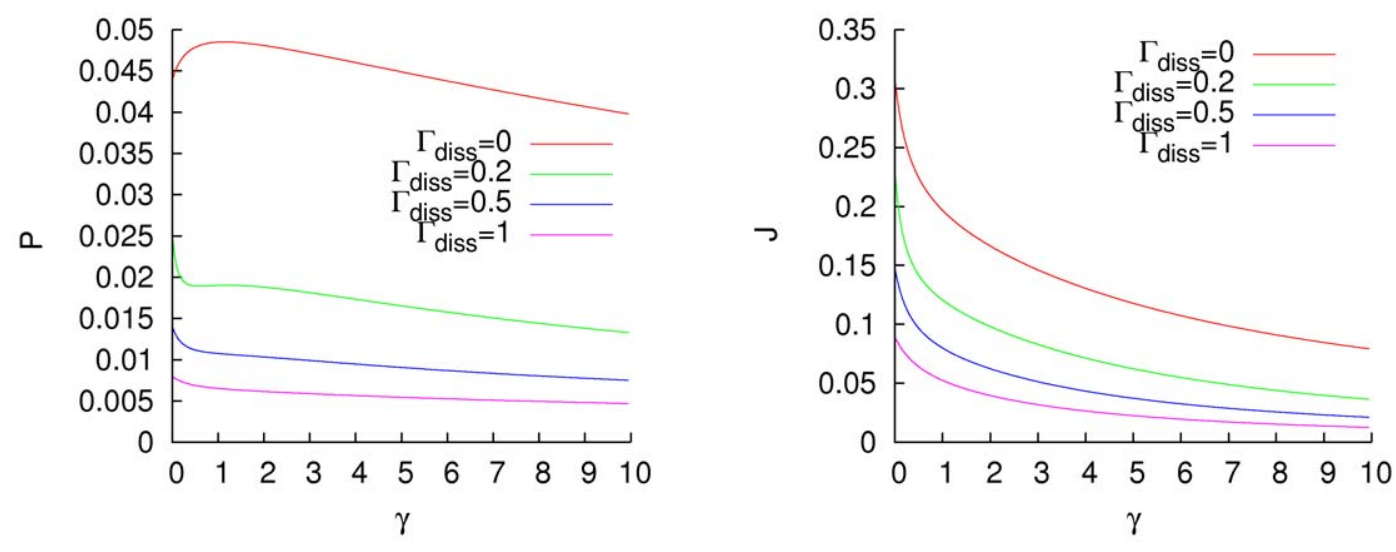

Figure 2. Excitation (left) and energy (right) fluxes for a homogeneous fully connected network, $\hbar \omega_{i}=\hbar g_{i j}=1 \mathrm{~cm}^{-1} \forall i, j$, with a thermal mean excitation number $n=100$, and different dissipation rates $\Gamma_{\text {diss }}$. Units of $\mathrm{cm}^{-1}$. doi:10.1371/journal.pone.0057041.g002 

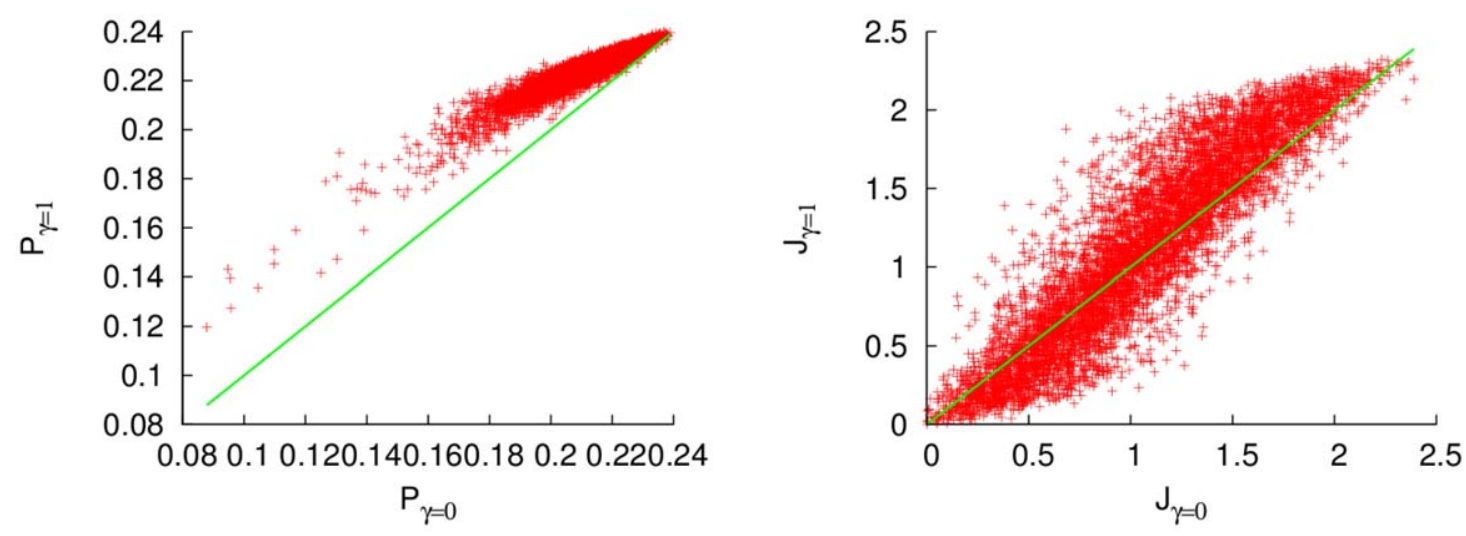

Figure 3. Excitation (left) and energy (right) fluxes for random Hamiltonians in the low-temperature regime $(n=1)$, for a dephasing ratio $\gamma=1$ as a function of the fluxes without dephasing. The green line separates the configurations with enhancement and depression of the transfer. Units of $\mathrm{cm}^{-1}$.

doi:10.1371/journal.pone.0057041.g003

efficiency comes from the differences between the one site energies and the couplings, which make the population of the third site the dominant component of Eq. (12).

As the FMO Hamiltonian is inferred from experimental spectroscopy data, it is subject to experimental uncertainty. To check a more complete scenario, we performed a Monte Carlo simulation. For this simulation, 7000 random Hamiltonians were generated, where each parameter $x$ corresponds to a Gaussian distribution with mean in the corresponding FMO parameter $x_{\mathrm{FMO}}$ and variance $\operatorname{var}=0.1 x_{\mathrm{FMO}}$. By this simulation we analyse random Hamiltonians with the same order of magnitude as that of the FMO.

For a low temperature bath, the results for the energy transfer are shown in Fig. 6. For small dephasing, most of the configurations are improved. This improvement is more important for configurations with low efficiency, and it is less for the most efficient ones. For higher values of $\gamma$, most of the configurations are degraded, principally only the ones with low efficiencies are enhanced. Again, this result is similar if we use the population of the sink as our index. This similarity can be understood by analysing Eq. (12). The energy flux depends on the population of the third site and on the coherences, modulated by the one site energy $\hbar \omega_{3}$ and the couplings $\hbar g_{3 i}$, respectively. If the one site energy of the third qubit is much higher than the couplings, the population of this site becomes the dominant term and both fluxes exhibit a similar behaviour.

In this section, we proved that for the FMO complex, it is possible to improve both the energy and the excitation fluxes. That is due, principally, to the fact that the FMO Hamiltonian is a configuration with a very low efficiency. If the Hamiltonian is modified in order to obtain a similar but more efficient one, this improvement reduces drastically.

\section{Conclusions}

In this paper, we analysed the energy transfer in quantum networks and its behaviour when external noise is added to the system. Special emphasis has been placed on networks that model a real photosynthetic light-harvesting system, the FMO complex from green sulfur bacteria. This analysis has been performed in a steady state scenario. In this regime, the evolution of the system is not conditioned on the arrival of individual excitations, and the energy flows across the system continuously.

From our analysis we can conclude the following:

- Even in the non-equilibrium steady state, there are time independent coherences in the system. These coherences can contribute both in a positive or negative way to the energy transfer.
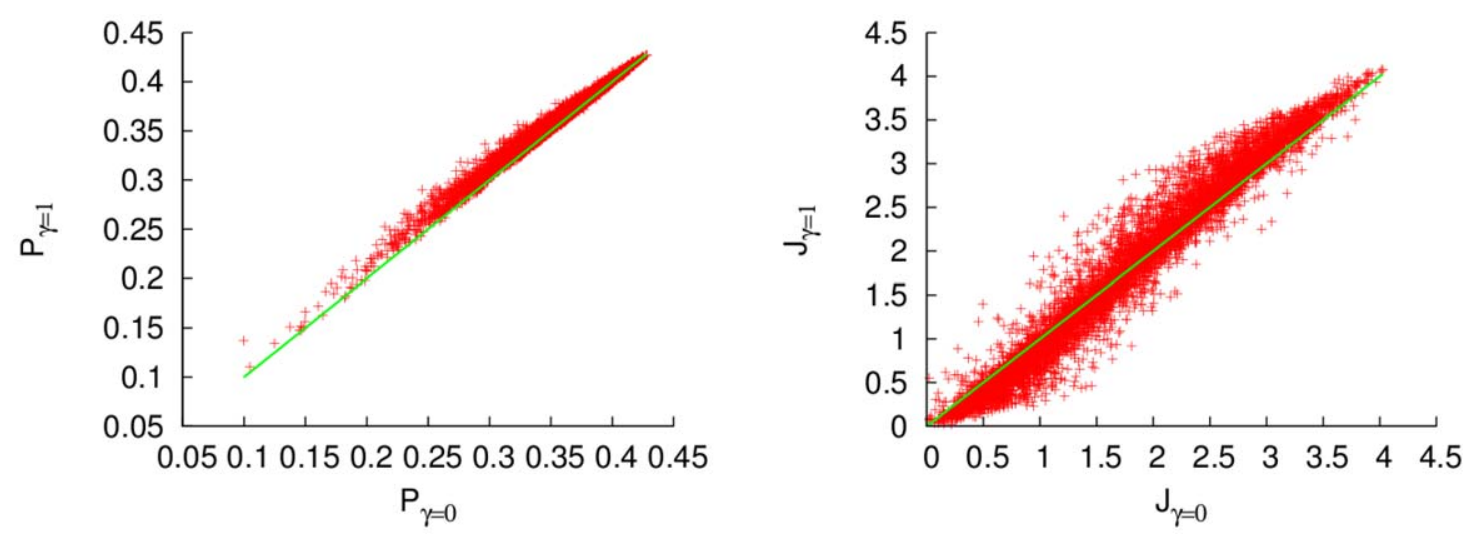

Figure 4. Excitation (left) and energy (right) fluxes for random uniformly distributed Hamiltonians in the low-temperature regime $(n=100)$, for a dephasing ratio $\gamma=1$, as functions of the fluxes without dephasing. The green line separates the configurations with enhancement and depression of the transfer. Units of $\mathrm{cm}^{-1}$. doi:10.1371/journal.pone.0057041.g004 

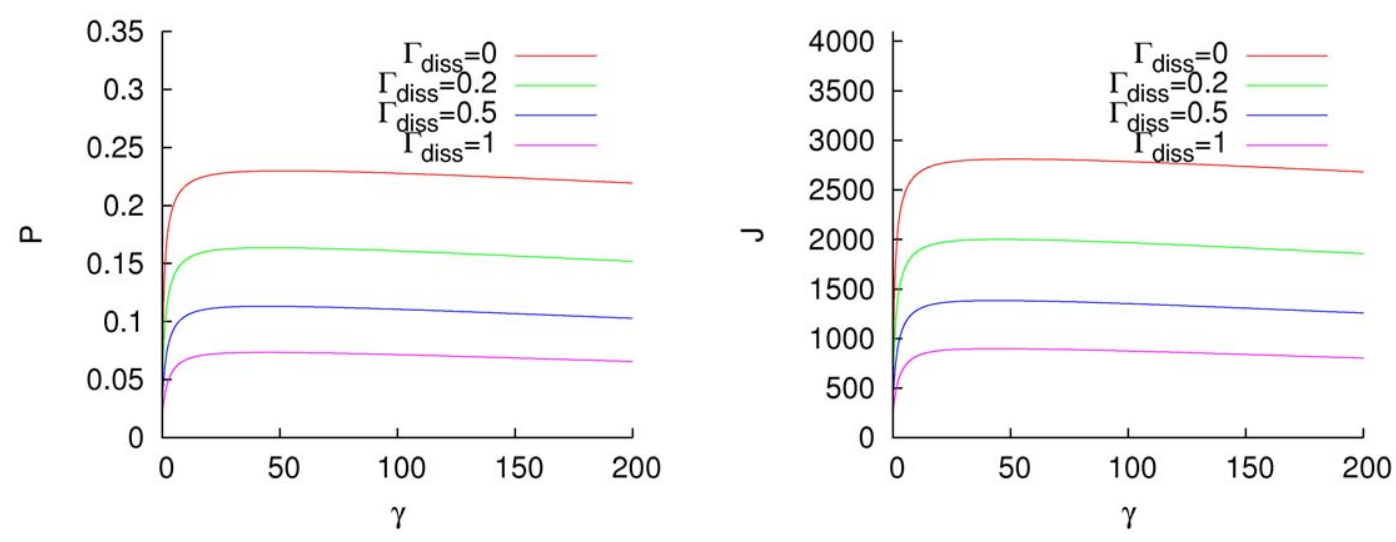

Figure 5. Excitation (left) and energy (right) fluxes from the network to the sink for the FMO Hamiltonian as functions of the dephasing ratio, $n=1$. Units of $\mathrm{cm}^{-1}$. doi:10.1371/journal.pone.0057041.g005

- The power of the system behaves in a very different way from the population transfer. That is due to the fact that the energy depends on the population but also on the coherences of the system.

- The population transfer is more amenable to improvement than the power. That means that even in the case in which the dephasing channel reduces the destructive interference in the system, it also reduces the coherences inside it, and that reduces the energy transfer in most cases.

- Also, the systems with low efficiency are more amenable to enhancement by a dephasing channel than the highly efficient ones. That is due to the fact that the interferences an be both destructive and constructive, and dephasing reduces both of them. Because of that, it can rarely improve a well performing configuration.

- Finally, systems that are in a high illumination regime are more stable under fluctuations due to the environmental interaction.

From the biological point of view, and keeping in mind that this is only a simple model far from the real photosynthetic scenario, we conclude that the energy transfer of the FMO complex can be enhanced by the addition of dynamical noise. On the other hand, this result is based on a single Hamiltonian inferred from empirical data. Our analysis shows also that if we slightly move from this Hamiltonian there are configurations that are better performing than the FMO. Also, we conclude that their energy transfer is not much enhanced by a dephasing channel. So, in order to design a highly efficient light harvesting system, there are two possibilities: it can have low efficiency in a pure coherent dynamic and can be improved under the effect of decoherence, or it can be just more efficient for the coherent dynamics in the first place. The optimal choice depends on the environmental situation and on the practical constraints, but in most of the cases analysed in this paper, the second choice is the optimal one.

\section{Acknowledgments}

The author would like to thank M. Tiersch, G.G. Guerreschi, and H.J. Briegel for useful conversations.

\section{Author Contributions}

Conceived and designed the experiments: DM. Performed the experiments: DM. Analyzed the data: DM. Contributed reagents/materials/ analysis tools: DM. Wrote the paper: DM.
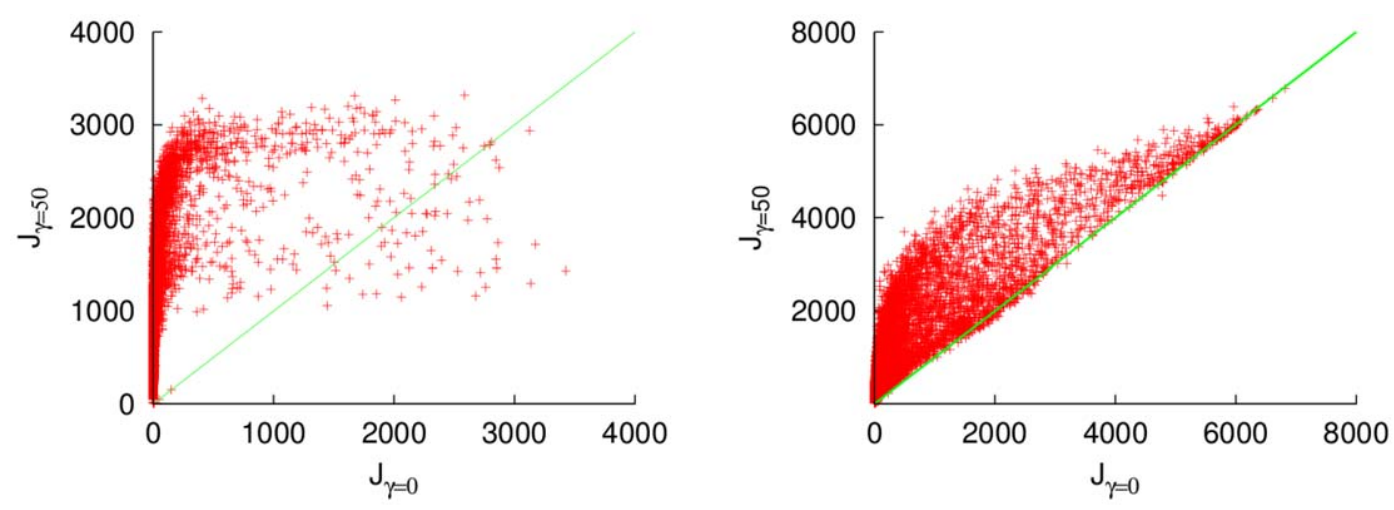

Figure 6. Energy flux for random Hamiltonians normally distributed around the FMO Hamiltonian, for $n=1$ (left) and $n=100$ (right). The $y$-axis represents the energy flux under a dephasing channel with $\gamma=50$ and the $x$-axis represents the energy flux without dephasing. The green line represents the space where both fluxes are equal. Units of $\mathrm{cm}^{-1}$. doi:10.1371/journal.pone.0057041.g006 


\section{References}

1. Engel GS, Calhoun TR, Read EL, Ahn TK, Mancal T, et al. (2007) Evidence for wavelike energy transfer through quantum coherence in photosyntetic systems. Nature 446: 782

2. Collini E, Wong CY, Wilk KE, Curmi PMG, Brumer P, et al. (2010) Coherently wired light-harvesting in photosynthetic marine algae at ambient temperature. Nature 463: 644 - 646.

3. Panitchayangkoon G, Hayes D, Fransted KA, Caram JR, Harel E, et al. (2010) Long-lived quantum coherence in photosynthetic complexes at physiological temperature. Proc Natl Acad Sci 107: 12766.

4. Plenio S, Huelga M (2008) Dephasing-assisted transport: quantum networks and biomolecules. J New Phys 10: 113019.

5. Mohseni M, Rebentrost P, Lloyd S, Aspuru-Guzik A (2008) Enviroment-assisted quantum walks in photosynthetic energy transfer. Journal of Chemical Physics 129: 174106

6. Olaya-Castro A, Lee CF, Olsen FF, Johnson NF (2008) Efficiency of energy transfer in a light-harvesting system under quantum coherence. PRB 78: 085115 .

7. Caruso F, Chin AW, Datta A, Huelga SF, Plenio MB (2009) Highly efficient energy excitation transfer in light-harvesting complexes: The fundamental role of noise-assisted transport. J Chem Phys 131: 105106.

8. Rebentrost P, Mohseni M, Kassal I, Lloyd S, Aspuru-Guzik A (2009) Environment-assisted quantum transport. NJP 11: 033003

9. Chin AW, Datta A, Caruso F, Huelga SF, Plenio MB (2010) Noise-assisted energy transfer in quantum networks and light harvesting complexes. J New Phys 12: 065002.

10. Wu J, Liu F, Shen Y, Cao J, Silbey RJ (2010) Efficient energy transfer in lightharvesting systems, I: Optimal temperature, reorganization energy and spatialtemporal correlations. NJP 12(105012).

11. Scholak T, de Melo F, Wellens T, Mintert F, Buchleitner A (2011) Efficient and coherent excitation transfer across disordered molecular networks. Phys Rev E 83(2): 021912.

12. Scholak T, Wellens T, Buchleitner A (2011) Optimal networks for excitonic energy transport. J Phys B: At Mol Opt Phys 44: 184012.
13. Mancal L, Valkunas T (2010) Exciton dynamics in photosynthetic complexes: excitation by coherent and incoherent light. NJP 12: 065044.

14. Brumer M, Shapiro P (2012) Molecular response in one photon absorption: Coherent pulsed laser vs. thermal incoherent source. Proc Natl Acad Sci 109: 19575.

15. Tiersch M, Popescu S, Briegel HJ (2012) A critical view on transport and entanglement in models of photosynthesis. Phil Trans R Soc A 370: 3771.

16. Manzano D, Tiersch M, Asadian A, Briegel HJ (2012) Quantum transport efficiency and Fourier's law. Phys Rev E 86: 061118.

17. Asadian A, Manzano D, Tiersch M, Briegel HJ (2013) Heat transport through lattices of quantum harmonic oscillators in arbitrary dimensions. Phys Rev E 87 : 012109 .

18. Blankenship RE, Tiede DM, Barber J, Brudvig GW, Fleming G, et al. (2011) Comparing photosynthetic and photovoltaic efficiencies and recognizing the potential for improvement. Science 332(6031): 805-809.

19. Cao JS (1997) A phase-space study of Bloch-Redfield theory. Journal of Chemical Physics 107: 3204.

20. Caruso F, Chin AW, Datta A, Huelga SF, Plenio MB (2010) Entanglement and entangling power of the dynamics in light-harvesting complexes. Phys Rev A 81(6): 062346.

21. Breuer F Petruccione HP (2002) The theory of open quantum systems. Oxford: Oxford University Press.

22. Rivas A, Plato AD, Huelga S, Plenio MB (2010) Markovian master equations: A critical study. J New Phys 12: 113032.

23. Adolphs T, Renger J (2006) How proteins trigger excitation energy transfer in the FMO complex of green sulfur bacteria. Biophys Journal 91(2778).

24. Jacobs K (2012) Quantum measurement and the first law of thermodynamics: The energy cost of measurement is the work value of the acquired information. Phys Rev E 86: 040106.

25. Linden N, Popescu S, Skrzypczyk P (2010) How small can thermal machines be? The smallest possible refrigerator. Phys Rev Lett 105(13): 130401. 